\title{
LA PREMIÈRE INTERVENTION EUROPÉENNE DANS L'EXPLOITATION DU CORAIL MAGHRÉBIN: LES CATALANS ET LES SICILIENS À TABARKA \\ (1446-1448)
}

\author{
PHILIPPE GOURDIN \\ Université Paris 12
}

(France)

\section{SOMMAIRE}

Introduction.- I. Les Catalans et les Siciliens à Tabarka: 1. La préparation de la campagne.- 2. Nombre et origine géographique des patrons et hommes d'équipage. 3. Une organisation légère et peu centralisée.- II. La cession de la pêche du corail aux Européens.- Conclusion.

\section{INTRODUCTION}

L'histoire de Tabarka, comme celle de sa voisine Marsacares (Masa'l Kharaz aujourd'hui la Calle en Algérie), est intimement liée à celle de ses riches bancs de coraux dont $\mathrm{Al}$ Içt'Akri vantait déjà les qualités au dixième siècle ${ }^{1}$; en 1542 , le fait est bien connu, l'île de Tabarka est cédée à certains membres des familles génoises des Grimaldi et des Lomellini et, pendant deux siècles jusqu'à sa prise par le souverain de Tunis, elle devient un comptoir génois abritant une ville de 1500 à 2000 habitants dont la

\footnotetext{
'"Tabarqa est une petite ville...Il y a en mer un gisement de corail qui est le seul que l'on connaisse au monde»; A. MIQUEL, La description du Maghreb dans la géographie d'Al-IÇt'Akri. dans "Revue de l'Occident musulman et de la Méditerranée", 1973-2, p. 233.

"Anuario de listudios Medievales". 27 (1997)
} 
prospérité repose, en grande partie, sur l'exploitation de corail'2. Moins connue, en revanche, est l'exploitation de ce même corail de Tabarka par les Catalans dès 1446, c'est à dire avant la concession de 1451 par laquelle le souverain hafside Othman accorde au Génois Clemente Cicero le monopole de la pêche du corail sur l'ensemble de la côte nord de son royaume, donnant ainsi naissance à plusieurs compagnies qui se succéderont jusqu'aux premières années du $\mathrm{XVI}^{\mathrm{e}}$ siècle et dont le siège se fixe à Marsacares ${ }^{3}$.

Comme cela arrive fréquemment, c'est un litige qui révèle la cession par le roi Othman du droit de la pêche du corail en «mer de Barbarie» à un certain Rafaël Vives, marchand catalan habitant Tunis; le 20 novembre 1446, les conseillers et prud'hommes de la ville de Cagliari, en Sardaigne, écrivent à leurs homologues de Barcelone pour leur faire part d'une plainte déposée par plusieurs pêcheurs de corail, originaires de Catalogne et de Sardaigne, pêchant en «mer de Barbarie» pour le compte de Rafaël Vives qui a reçu du souverain de Tunis la ferme de la pêche de corail; ces pêcheurs se plaignent, en effet, de la part, trop élevée à leur goût, du corail qu'ils doivent remettre à Rafaël, et veulent qu'elle soit abaissée du tiers au dixième des coraux pêchés; et, pour appuyer leur demande, ils expliquent qu'en plus du risque de la mer, ils doivent affronter un environnement difficile; ils suggèrent en outre, que l'on fasse appel au frère de Rafaël, Luis Vives qui réside à Barcelone, probablement le chef de la famille, pour qu'il intervienne auprès de son frère; mais, dans leur réponse, les conseillers de Barcelone annoncent le refus de Luis qui se contente de garantir aux pêcheurs sardes des conditions analogues à celles qui sont consenties aux Catalans ${ }^{4}$.

Dans ces deux lettres, il n'est pas fait mention de Tabarka, mais seulement des «mers de Barbarie», tandis qu'au même moment, de 1446 à 1448, certains notaires de Trapani, en Sicile, instrumentent des actes qui

'F. PODESTÀ, L'isola di Tabarca e le pescherie di corallo nel mare circostante, dans "Atti della Società ligure di Storia Patria", XIII (1884), pp. 1005-1044.

${ }^{3} \mathrm{P}$. Gourdin, Emigrer au $X V^{\prime}$ siècle. La communauté ligure des pêcheurs de corail de Marsacares. I. Etude de la population et des modalités de départ, dans "Mélanges de l'École française de Rome". Moyen Âge-Temps Modernes, 98, 1986-2, pp. 543-605; II. Vie quotidienne, pouvoirs, relations avec la population locale, dans "Mélanges de l'École française de Rome". Moyen Âge, 102, 1990-2, pp. 131-171.

${ }^{4}$ Archivio Historico de la Ciudad de Barcelona (Archives municipales de Barcelone), Lett. Origin., année 1446, 20 novembre et 14 décembre; Les deux lettres sont publiées dans: A DE CAPMANY Y DE MONTPALAU, Memorias historicas sobre la marina, el comercio y las artes de la antigua ciudad de Barcelona, Madrid, 1779, II, pp. 267-269. 
témoignent de la venue de pêcheurs in maribus de Tabarkii ${ }^{5}$ sans faire référence à Rafaël Vives ${ }^{6}$; mais il est certain que ces pêcheurs travaillent pour notre marchand, ou son éventuel successeur, car le bénéficiaire de la ferme a le monopole de l'exploitation. Le centre d'exploitation du corail s'est donc fixé à Tabarka, alors que les Génois qui reprendront la concession en 1451 s'installeront à Marsacares.

C'est cette première expérience européenne de pêche de corail africain $^{7}$ que je voudrais tout d'abord tenter de cerner en utilisant la cinquantaine d'actes notariés provenant des archives de Trapani ${ }^{8}$. Qui sont les pêcheurs? Quelle est leur organisation? Comment vivent-ils...? D'utiles comparaisons avec les compagnies génoises de Marsacares ou avec la situation sicilienne permettront parfois de pallier la relative pauvreté de ces sources. Ensuite, je poserai la question qui me paraît essentielle: Pourquoi

\footnotetext{
${ }^{5} \mathrm{~A}$ ma connaissance, les premières références de la venue de ces pêcheurs à Tabarka sont dues à: C. TRASSELl, Sull'arte in Trapani nel'400, Trapani, 1948, p. 40; reprises et développées par G. ET H. BRESC, Il corallo siciliano nel Mediterraneo, dans "La Fardeliana", 2-3 (1982), pp. 39-49; G. BRESC-BAUTIER, Le corail sicilien, dans L'homme méditerranéen é la mer ("Actes du troisième congrès international d'études des cultures de la Méditerranée Occidentale" (Jerba, avril 1981), Tunis, 1985, p. 183; H. BRESC, Un monde méditerranéen, économie et société en Sicile, Rome, I, 1986, p. 226. F. Podestà, en revanche, ignore la venue des Catalans et Siciliens à Tabarka: F. PODESTA, L'isola di Tabarca, op. cit.

${ }^{6}$ Seulement deux actes provenant des archives de Trapani font référence au corail et à Rafaël Vives; ils concernent un litige entre le marchand et les pêcheurs; c'est probablement ce litige qui est à la source de la lettre des conseillers de Cagliari : Archivio di Stato di Trapani (AST), notaire Francesco Milo, reg. 8626, 10 et 18 juillet 1446, $\mathrm{f}^{\circ} 329 \mathrm{r}-330 \mathrm{v}$.

${ }^{7}$ Plusieurs auteurs affirment que les Pisans ont exploité le corail maghrébin dès le XII siècle; par exemple L. DE MAS LATRIE, Documents sur l'histoire de l'Algérie et de l'Afrique septentrionale pendant le Moyen Âge, dans "Bibliothèque de l'École des Chartes", 10 (1848-49), p. 135, (repris par de nombreux auteurs dont F. BRAUDEL, La Méditerranée à l'époque de Philippe II. nouv. éd. Paris, 1979, I, p. 197; ainsi que H.-R. IDRIS, La Berbérie orientale sous les Zirides, X-XIIe siècles, Paris, 1962, p. 680) qui affirme que les Pisans prennent Tabarka en 1140 et y exploitent les bancs de coraux; en fait L. de Mas Latrie se réfère à deux auteurs antérieurs: FANUCCI, Storia dei tre popoli marittimi dell'Italia, Veneziani, Genovesi e Pisani, Pise, 1817, II, p. 48; et RonCIONI, Istorie Pisane, Florence, 1844, I, p. 255, et ne reprend pas ces informations dans son principal ouvrage: L. DE MAS LATRIE, Traités de paix et de commerce et documents divers concernant les relations des chrétiens avec les Arabes de l'Afrique septrionale, 2 vol., Paris, 1866. Selon Fanucci, le traité de 1157 entre Pise et Tunis comprend un article sur cette exploitation du corail; mais le texte du traité (dans sa version latine ou dans la traduction italienne de sa version arabe) ne parle ni de corail ni de Tabarka; L DE MAS LATRIE, op. cit., II, pp. 23-26; F. PODESTȦ, L'isola di Tabarca, op. cit. p. 1013, a déjà mis en doute cette information.

${ }^{8} \mathrm{Ces}$ actes se trouvent dans les registres des notaires suivants: AST, Durduglia de Durduglia, reg. 8606, année 1446-1447; Giacomo Miciletto, reg 8592 et 8593 , années 14451447; Francesco Milo, reg. 8626, 8627, 8628 et 8629, années 1445-1449; Giovanni Scanatello, reg. 8554, années 1447-1449; Benedetto Trusello, reg. 8680, année 1446-1447.
} 
le souverain fait-il appel à des Européens, Catalans en 1446, puis Génois en 1451, alors qu'une tradition locale fort ancienne de pêche de corail est attestée par les auteurs musulmans?

\section{Les CATAlans et les Siciliens à TABARKA}

La première exploitation européenne du corail de Tabarka semble avoir été de courte durée puisque la cinquantaine d'actes recueillis aux Archives de Trapani ont tous été instrumentés entre juillet 1446 et juillet 1448; on observe, en outre, une forte irrégularité de leur distribution, avec une concentration d'actes écrits pendant le printemps et l'été 1447 (41 actes, soit plus de $80 \%$ du total), contre sept en 1446 et deux en 1448; la plupart de ces actes notariés sont des contrats pour le recrutement des équipages ou le financement de la pêche qui rendent compte de préparatifs annonçant un départ proche, tandis que d'autres, moins nombreux, clôtures de compte ou litiges, se réfèrent à une campagne de pêche terminée.

Le premier acte connu, datant du 18 juillet 1446, concerne un litige sur une vente de corail, ce qui signifie que la campagne de pêche a déjà eu lieu; si l'on se réfère à l'année 1447 pour laquelle la documentation est plus abondante, on constate que le mois d'avril correspond aux plus nombreux départs; de même, le dernier acte connu, datant du 6 juillet, 1448, est un accord de financement pour une campagne future; on ignore, bien sûr, si la campagne eut lieu, mais, si l'on reprend le modèle de l'année 1447, le retour a dû s'effectuer en septembre $1448^{9}$; dans ces conditions, l'exploitation aurait duré un peu plus de deux ans en s'étalant sur trois étés.

Les pêcheurs se sont-ils tous rendus à Tabarka? C'est certain, même si les sources ne l'écrivent pas toujours de façon explicite; en effet, plusieurs expressions sont utilisées pour indiquer les lieux de pêche; le nom de Tabarka revient dans la moitié des contrats, tandis que dans l'autre moitié, les notaires emploient une formule plus vague in partibus Barbarie ou ad partes barbaricas ou encore, dans un cas in maribus Thunisii ${ }^{10}$; le notaire Miciletto emploie l'expression la plus précise: in partibus barbaricis, in loco

\footnotetext{
${ }^{9}$ Premier acte connu: Milo, 8626, 18 juillet $1446, \mathrm{f}^{\circ} 329$; dernier acte connu: Milo, 8628 , 6 juillet $1448, \mathrm{f}^{\circ} 315 \mathrm{r}$; cet acte est cassé le 30 novembre 1452, une date tardive qui est peut-être le signe de difficultés.

${ }^{10}$ In maribus Thunisii, dans Milo, 8628, 6 juillet 1448, $\mathrm{f}^{\circ} 315 \mathrm{r}$.
} 
nuncupato Tabarcall; en revanche, aucun autre nom de lieu n'apparaît, ce qui fait indubitablement de Tabarka le centre de l'exploitation ${ }^{12}$.

Il ne faut pas s'étonner de la venue à Tabarka de Catalans et de Siciliens, car depuis le XIII ${ }^{e}$ siècle et la conquête de la Sicile par la Couronne d'Aragon, les côtes du Maghreb leur sont devenues familières soit pour le commerce soit pour la course ${ }^{13}$; témoins de l'ancienneté de la connaissance des lieux par les Européens, les premiers portulans connus mentionnent tous Tabarka, qu'il s'agisse du Liber de existencia rivierarum pisan, ou du Conpasso da navigare génois ${ }^{14}$; quant aux cartes nautiques des $\mathrm{XIV}^{\mathrm{e}}$ et $\mathrm{XV}^{\mathrm{e}}$ siècles, elles inscrivent toutes Tabarka en rouge, la couleur des ports les plus importants ${ }^{15}$. En fait, au milieu du $X^{\mathrm{e}}$ siècle, l'ancienne ville et le port n'existent plus depuis longtemps ${ }^{16}$, mais Tabarka demeure le meilleur mouillage entre Bône et Bizerte et figure, à ce titre, sur les cartes.

Le choix de Tabarka répond en outre à un souci de sécurité; les pêcheurs chrétiens ont besoin d'un lieu où ils peuvent entreposer le précieux corail et se protéger d'une population nomade peu nombreuse mais dont la réputation est bien établie ${ }^{17}$; ne pouvant $s^{\prime}$ installer dans la forteresse de Marsacares, ils trouvent à Tabarka un site idéal grâce à son île située à

\footnotetext{
"Miciletto, 8593, $1^{\text {er }}$ mars $1447, f^{\circ} 64 r$.

${ }^{12}$ Marsacares apparait une fois comme port d'embarquement d'une caisse de corail; des procurateurs sont nommés pour récupérer auprès d'un marchand majorquin 10 doubles restant à devoir sur le transport de cette caisse sur une barque appartenant à un Valencien; Milo, 8629, 27 août $1449, \mathrm{f}^{\circ} 252 \mathrm{r}-253 \mathrm{v}$.

${ }^{13}$ On peut se référer à C.-E. DufourCQ, L'Espagne catalane et le Maghrib aux XIII' et XIV siècles, Paris, 1965, en particulier la carte de la navigation et des routes en fin de volume.

${ }^{14} \mathrm{P}$. GaUtIER DalChÉ, Carte marine et portulan au XII" siècle. Le "Liber de existencia rivierarum et forma maris nostri Mediterranei", Rome, 1995, p. 120 ; B. R. MOTZO, Il Compasso da navigare, opera italiana della metà del secolo XIII, Cagliari, 1947, p. 74.

${ }^{15}$ Depuis le «modèle» de la «pisane», deux couleurs différencient les ports: le rouge pour les plus importants et le brun pour les autres; de nombreuses cartes sont reproduites dans: $M$. MOLLAT DU JOURDIN et M. DE LA RONCIĖRE, Les portulans, Fribourg, 1984.

${ }^{16}$ II n'y a pas de rupture entre l'antique Thabraka et la ville musulmane qui connaît son plus grand développement à l'époque aghlabide et au début de l'époque fatimide grâce aux échanges avec Al Andalus; Idrissi note encore, au XIIe siècle, la présence de marchands andalous, puis c'est le déclin et l'abandon, peut-être dès le XIIIe siècle. Cf. P. Gourdin, Tabarka, Histoire et archéologie d'un comptoir européen en terre africaine $\left(X V^{\prime}-X V I I I^{\prime}\right)$, à paraître.

${ }^{17} \mathrm{Au}$ XIVe siècle, Ibn Battûta souligne les dangers de la route qui relie Bône à Tunis; Les marchands qui font partie de la caravane préfèrent se rendre à Tunis en bateau plutôt que de risquer de se faire rançonner; IBN BATTÙTA, Voyages, trad. C. Defréméry et B.R. Sanguinetti, Paris, nouv. éd. 1990 , II, p. 84.
} 
quelques centaines de mètres de la côte, une distance suffisante pour offrir une défense naturelle; mais les pêcheurs ne peuvent s'y murer car ils doivent obligatoirement chercher l'eau potable sur la terre ferme; en fait, ils vivent dans leurs barques, choisissant leur mouillage en fonction du vent et de l'état de la mer, et l'île leur sert de base de stockage et de lieu de repli en cas d'insécurité.

Le corail se pêche à l'aide d'un «engin» (ingenium), croix de Saint André lestée ayant à ses extrémités des filets (redacia); l'engin est descendu à une profondeur pouvant atteindre une centaine de mètres à l'aide d'un filin (sarcia) et racle les rochers; l'exercice demande de la force et une bonne connaissance des lieux favorables; il n'existe pas, à proprement parler, de saison de pêche, dans la mesure où le corail peut être pêché toute l'année, été comme hiver; plusieurs barques s'apprêtent à partir à Tabarka en octobre et décembre 1446; l'une d'elles revient en février et repart en avril $1447^{18}$; il n'en reste pas moins que les départs les plus nombreux s'effectuent au printemps et au début de l'été avec un retour prévu en septembre ${ }^{19}$; il est certain que l'état de la mer est plus favorable en été et que les prises sont plus nombreuses.

\section{La préparation de la campagne}

Les barques séjournent donc en Barbarie un temps relativement court, de deux à cinq mois; mais la préparation de la campagne peut se révéler longue et fastidieuse; le patron doit d'abord constituer son équipage; l'un d'eux, Bartholomeus de Avillaneda a besoin de trois mois pour recruter neuf hommes ${ }^{20}$; le patron doit aussi trouver un financement pour acheter à l'avance l'équipement nécessaire à la navigation et à la campagne de pêche (sarcia et redacia sont utilisés en grande quantité parce que souvent perdus ou détériorés); enfin le patron fait des provisions de bouche et donne une avance à ses hommes. Tout doit être apporté à Tabarka et les barques

\footnotetext{
${ }^{18}$ Trois barques en octobre 1446: Durduglia, 8606, 4 octobre $1446, f^{\circ} 45 v-46 v$; une barque en décembre 1446: Miciletto, 8592, 29 décembre 1446, $\mathrm{f}^{\circ} 47 \mathrm{r}$. Le patron Sagoya effectue deux campagnes d'hiver: Durduglia,8606, 4 octobre 1446, $\mathrm{f}^{\circ} 46 \mathrm{v} ; 14$ mars $1447, \mathrm{f}^{\circ} 125 \mathrm{v}$.

${ }^{19}$ Onze départs de barque en avril, deux en mai, deux en juin, deux en juillet; les retours sont généralement prévus en septembre.

${ }^{20}$ Le premier homme est recruté le 15 février pour un départ qui aura lieu fin avril ou début mai 1447.
} 
transportent donc du vin, des fromages, des cordages et même, lorsqu'il reste de la place, des produits interdits de vente dans les pays musulmans, comme le fer, et dont les bénéfices attendus sont substantiels ${ }^{21}$.

Généralement le patron n'a pas la surface financière suffisante et fait appel à un ou plusieurs tiers qui lui prêtent de l'argent ad modum parcium, à la part; le financier devient associé et participe au produit de la pêche en fonction de son apport, au même titre que les autres sociétaires, patron et hommes d'équipage. Théoriquement, tous les systèmes de calcul «à la part» sont possibles; il suffit, pour cela, que tous les associés se mettent d'accord sur la valeur de la part et sur la répartitions des parts; mais certains usages se sont peu à peu généralisés; le patron Johannes Sagoya recrute quatre hommes en mars et avril 1447; ensemble, ils constituent une société de sept parts, trois pour Sagoya et une pour chacun des hommes ${ }^{22}$; la valeur de la part semble fixée de façon coutumière à 6 onces $^{23}$, c'est à dire, approximativement, le prix d'une barque équipée mais sans provision et matériel de campagne ${ }^{24}$; la barque représente donc un apport de capital équivalent à une part; la septième part correspond au numéraire nécessaire pour financer l'opération et peut, en partie ou en totalité, être rétrocédée à un financier au prorata de son apport. Au total, sur sept parts, cinq rémunèrent le travail et deux le capital (une pour la barque et l'autre pour l'apport en numéraire) et la part est évaluée à 6 onces.

D'autres équipages sont recrutés sur la base du salariat; les neuf hommes recrutés par Bartholomeus de Avillaneda sont tous des salariés; sept reçoivent un salaire de 21 tari, les deux autres recevant 18 et 12 tari; en

\footnotetext{
${ }^{21}$ Un patron corailleur transporte 4,5 salmes de vin dans sa barque: Milo, 8627, 20 mai $1447, \mathrm{f}^{\circ} 264 \mathrm{v}$; Bartholomeus de Avillaneda transporte 6 cantares de fer pisan: Milo, 8627, 18 avril $1447, f^{\circ} 214 \mathrm{r}$. D'autres transports de victuailles sont effectués par des barques ordinaires : 5 salmes de vin et 4 cantares de fromage sur la barque de Julianus de Aurichio: Milo, 8627, 12 et 13 juin 1447, $\mathrm{f}^{\circ} 299 \mathrm{v}$ et $302 \mathrm{r}$; le Maltais Micael Grecu assure une autre livraison de vin : Durduglia, 8606, 21 août $1447, \mathrm{f}^{\circ} 247$.

${ }^{22}$ Durduglia, 8606, 14 mars et $1^{\text {tr }}$ avril $1447, \mathrm{f}^{\circ} 125 \mathrm{v}$.

${ }^{23}$ Cette valeur de 6 onces par part est, en effet, rappelée dans plusieurs actes: Miciletto, $8593,1^{\text {er }}$ mars $1447, f^{\circ} 64 \mathrm{r} ; 15$ avril, $f^{\circ} 78 \mathrm{v} ; 13$ et 15 mai $1447, f^{\circ} 88 \mathrm{r}$ et $89 \mathrm{v}$.

${ }^{24}$ Une telle barque est vendue 5 onces: Trussello, 8680, 21 juin $1447, f^{\circ} 149 \mathrm{v}$; une barque munie de ses provisions de campagne peut valoir le double ou plus; la moitié d'une telle barque est vendue 7,5 onces: Trussello, 8680,7 juillet $1446, f^{\circ} 154 \mathrm{v}$.
} 
outre, le patron leur fournit la nourriture et la boisson et leur avance l'équivalent d'un mois de salaire ${ }^{25}$.

\section{Nombre et origine géographique des patrons et hommes d'équipage}

Les sources permettent de dénombrer 28 patrons de barque, 31 barques et 29 hommes d'équipage. Le terme de patron est ambigu; en général il désigne l'homme qui conduit la barque, mais il arrive qu'il en soit seulement le propriétaire et qu'il la confie à un raïs; d'autre part, certains patrons possèdent plusieurs barques qui pêchent de concert. Normalement, l'équipage d'une barque de corailleurs se compose de quatre hommes qui, en Provence, sont appelés properio, proherio, mejerio et trelherio ${ }^{26}$; on retrouve ce chiffre de quatre hommes par barque dans les autres régions méditerranéennes productrices de corail à la fin du Moyen Âge, en Catalogne, en Ligurie, en Sardaigne, dans le Royaume de Naples comme plus tard à Marsacares ${ }^{27}$; pour une raison qu'il est difficile d'expliquer, les barques siciliennes sont souvent plus grandes et les équipages se composent fréquemment de cinq ou six hommes; cette singularité sicilienne peut éventuellement s'expliquer par le fait que les barques utilisées pour la pêche du corail servent à d'autres activités, en particulier la pêche au thon et qu'elles sont, pour cette raison munies de quatre à six paires de rames ${ }^{28}$.

L'exception sicilienne s'exporte à Tabarka; les quelques mentions de ventes de barques ou de parts de barque destinées à la pêche sur les côtes de Barbarie concernent toutes des embarcations de cinq et six paires de

\footnotetext{
${ }^{25}$ Consalvus Amatus de Séville et Bartholomeus Vinicianus s'engagent auprès du patron Avillaneda pour corailler in partibus de Barbarie sur l'une de ses deux barques: Consalvus recevra un salaire mensuel de 12 taris et Bartholomeus de 18 taris; le patron fournira en outre victu et potu; les hommes reçoivent l'équivalent d'un mois avant le départ et le reste au retour; Milo, 8627, 3 avril 1447, $\mathrm{f}^{\circ}$ 199r.

${ }^{26} \mathrm{G}$. LAVERGNE, La pêche et le commerce du corail à Marseille aux XIV" et XV' siècles, dans "Annales du Midi" (1952), p. 201.

${ }^{27} \mathrm{Ce}$ nombre de quatre hommes par barque n'est valable que pour la fin de la période médiévale; en effet, les barques des XVII ${ }^{\mathrm{e}}$ et $\mathrm{XVIII}{ }^{\mathrm{e}}$ siècles auront généralement des équipages plus importants. De son côté, Ibn Hawqal parle d'équipages de vingt hommes à Marsacares au Xe siècle: IBN HAWQAL, Configuration de la terre, trad. de Slane, dans "Journal Asiatique" (1842), p.181

${ }^{28} \mathrm{Contrairement}$ à ce qu'affirme G. Bresc-Bautier, les barques des corailleurs trapaniotes ne contiennent pas des équipages de deux à quatre hommes, mais de quatre à six hommes; $G$ BRESC-BAUTIER, Le corail sicilien, op. cit., p. 183.
} 
rames $^{29}$, et les quelques équipages complets qui peuvent être reconstitués se composent de cinq hommes; ainsi le patron Bartholomeus de Avillaneda engage neuf hommes entre le 15 février et le 19 avril 1447 pour équiper ses deux barques qui, probablement, disposent chacune de cinq paires de rames $^{30}$. Si l'on accepte ce chiffre moyen de cinq hommes par barque, ce sont au minimum 150 pêcheurs qui se sont rendus à Tabarka de 1446 à 1448 , certains y revenant plusieurs fois ${ }^{31}$; mais il est évident que le chiffre réel doit être bien supérieur, ne serait-ce qu'en raison du silence des notaires de Trapani sur les pêcheurs sardes, ou catalans vivant en Sardaigne, que nous révèle la lettre des conseillers de Cagliari à ceux de Barcelone ${ }^{32}$. On peut comparer ce chiffre à celui qui a été calculé pour la communauté de pêcheurs de Marsacares ${ }^{33}$; d'après les sources ligures, 53 barques et 212 pêcheurs sont effectivement partis corailler en 1456 quatre ans après le début de la concession, mais on a pu, en utilisant différents critères, évaluer le nombre total de pêcheurs à 320 . La communauté de Tabarka atteint très certainement un chiffre moins élevé; dès 1447 , année où les sources sont les plus nombreuses, les pêcheurs répertoriés sont déjà $125^{34}$, chiffre relativement élevé qui suggère que le début de la concession de Rafaël Vives pourrait être antérieure à 1446 .

\footnotetext{
${ }^{29}$ Trois contrats de vente de barques qui vont pêcher à Tabarka sont connus; une barque de cinq paires de rames est vendue avec tout l'équipement nécessaire à la pêche au prix de 5 onces; La moitié de la seconde, une barque de six paires de rames, est vendue 7,5 onces: Trussello, 8680,21 juin $1447, \mathrm{f}^{\circ} 149 \mathrm{v}$ et 7 juillet $1447, \mathrm{f}^{\circ} 154 \mathrm{v}$; enfin peu avant son départ, le patron Sagoya vend un quart de sa barque au prix de 5 onces 22 taris et 10 grains; le prix comprenc l'équipement nécessaire à la navigation et à la pêche et, également, les provisions destinées à la campagne: Milo, 8627, 15 avril, $f^{\circ} 209 r$.

${ }^{30}$ Miciletto, 9492, 15 février 1447, fo $61 \mathrm{r}$ et v; 19 avril 1447, $\mathrm{f}^{\circ} 80 \mathrm{r}$; Milo, 8627, 29 mars $1447, \mathrm{f}^{\circ} 189 \mathrm{r} ; 3$ avril $1447, \mathrm{f}^{\circ} 199 \mathrm{r} ; 13$ avril $1447, \mathrm{f}^{\circ} 205 \mathrm{r}$. D'autre part le patron Nicolaus de Manso forme une association avec quatre hommes qui l'accompagnent dans sa barque: Durduglia, 8606, 4 octobre 1446, $\mathrm{f}^{\circ}$ 46r; Johannes Sagoya fait de même avec quatre marins: Durduglia, 8606, 14 mars et $1^{\text {er }}$ avril 1447, fo $125 \mathrm{r}$ et 27 avril, $\mathrm{f}^{\circ} 154$.

${ }^{31}$ Le patron Johannes Sagoya part une première fois après le 4 octobre 1446; il est de retour le 15 février 1447, date à laquelle l'acte précédent est cassé; il prépare un nouveau départ en mars-avril 1447: Durduglia, 8606, 4 octobre 1446 cassé le 15 février 1447, f ${ }^{\circ} 46 \mathrm{v}$; 14 mars et $1^{\text {er }}$ avril $1447, \mathrm{f}^{\circ} 125 \mathrm{r}$ et 27 avril $1447, \mathrm{f}^{\circ} 154 \mathrm{r}$.

${ }^{32}$ Sauf en cas de litige; ce qui est le cas pour trois patrons catalans habitant Cagliari: Milo, 8626,18 juillet $1446, \mathrm{f}^{\circ} 329 \mathrm{r}$.

${ }^{33} \mathrm{P}$. Gourdin, Émigrer au XV' siècle, I, op. cit. pp. 580-585.

${ }^{34}$ Le décompte année par année des pêcheurs présents à Tabarka est le suivant: 1446: 10 barques et 50 pêcheurs; 1447: 25 barques et 125 pêcheurs; 1448: 2 barques et 10 pêcheurs; la campagne d'hiver 1446-47 est comptabilisée dans les deux années.
} 


\section{Carte $n^{0} 1$}

Lieu de résidence et origine géographique

des patrons de barque

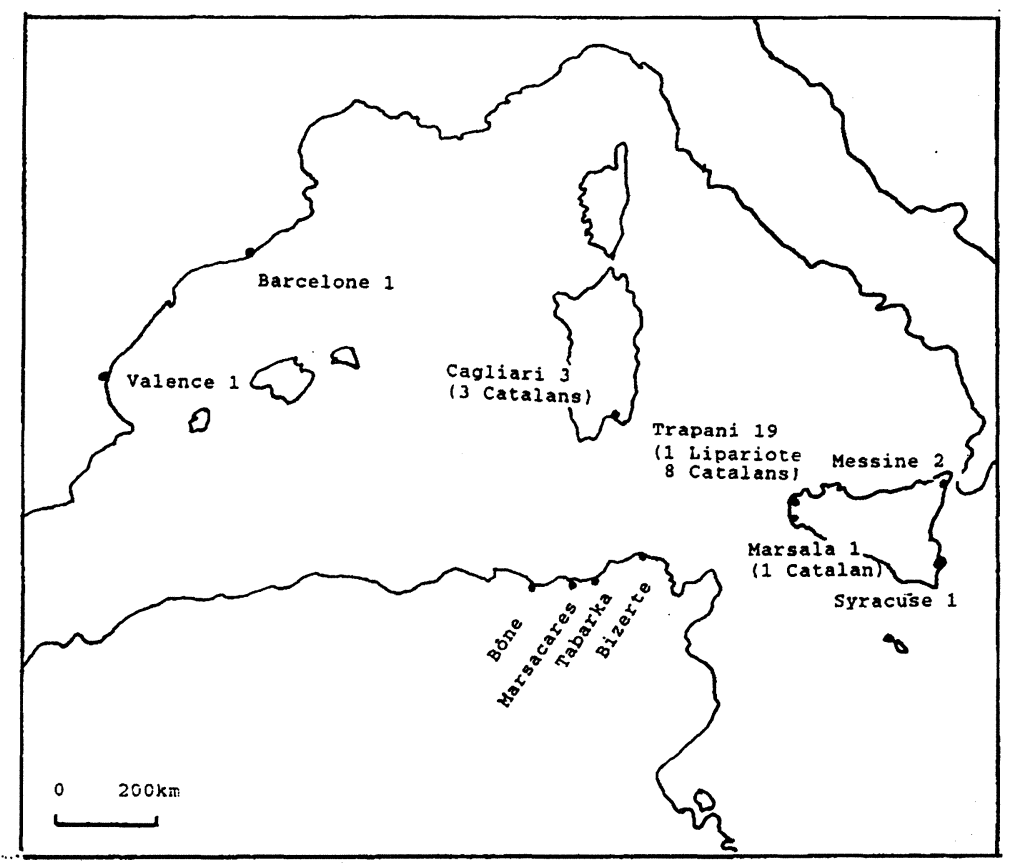

Légende: Trapani 19 (8 catalans)

signifie que. parmi les 19 patrons qui résident à Trapani, 8 sont qualifiés de Catalans ou portent un nom catalan 
L'origine de pêcheurs est extrêmement variée; les 28 patrons dont les noms sont connus, habitent, dans leur grande majorité $(68 \%)$ le territoire de Trapani (carte $n^{\circ} 1$ ); seuls 9 patrons résident habituellement dans une autre ville, un Barcelonais, un Valencien, ainsi que quatre Catalans qui ont émigré, trois à Cagliari en Sardaigne et un à Marsala, près de Trapani; à ces six Catalans, il faut ajouter trois Siciliens, deux de Messine et un de Syracuse. Les 19 autres patrons sont qualifiés de habitator terre Drepani, mais, parmi eux, neuf sont des immigrants de date plus ou moins récente; c'est le cas naturellement de Jacobus de Lipari, mais aussi de 8 patrons dont les noms (Insaglatu, Insumino...) ou les prénoms (Jaime, Lois, Pere...) indiquent une origine catalane pas toujours clairement exprimée. Au total, la moitié des patrons (14 sur 28) sont catalans ou d'origine catalane, quatre viennent d'autres parties de la Sicile ou des îles; il n'est pas exclu que, parmi les dix patrons restants, certains soient d'immigration récente mais leur lieu d'origine est inconnu.

L'étude de l'origine géographique des hommes d'équipage fait apparaître une dispersion encore plus grande (carte $n^{\circ} 2$ ); il faut parfois, comme pour les patrons, distinguer la ville de résidence de la ville d'origine; mais il existe, également, une autre catégorie de marins, apparemment sans résidence fixe, dont le nom seul révèle la provenance; ces marins, généralement originaires de ports et côtes de la Méditerranée occidentale s'embarquent au gré des opportunités. Sur 29 hommes d'équipage connus, neuf seulement résident à Trapani; parmi eux, un s'avoue Valencien et trois autres portent un nom catalan; deux hommes résident dans des localités proches de Trapani, le premier à Marsala et le second, un Provençal, à Monte San Giuliano (actuelle Erice); cinq viennent d'autres villes de Sicile, un de Syracuse, un autre, un Maltais, de Catane et trois de Messine (dont deux qui portent un nom catalan); restent onze hommes originaires de toutes les régions de la Méditerrnanée occidentale et, même, de l'Atlantique: trois Barcelonais, un Valencien, un Aragonais, un Provençal, un Ligure de la Rivière de Gênes, un Vénitien, un Sévillan, un Gascon et enfin, un homme originaire de Flandre. On retrouve une forte présence catalane, quoique à un degré moindre que pour les patrons; en effet, onze marins sont des sujets de la Couronne d'Aragon (trois Barcelonais, un Valencien et un Aragonais); mais la géographie du recrutement des hommes d'équipage dépasse les régions traditionnelles de production du corail; à la Catalogne, la Provence et la Ligurie s'ajoutent les îles proches de la Sicile comme les Lipari et Malte, deux foyers d'intenses activités liées à la mer, ainsi que quelques 


\section{Carte $\mathrm{n}^{\circ} 2$}

Lieu de résidence et origine géographique des hommes d'équipage (patrons exclus)

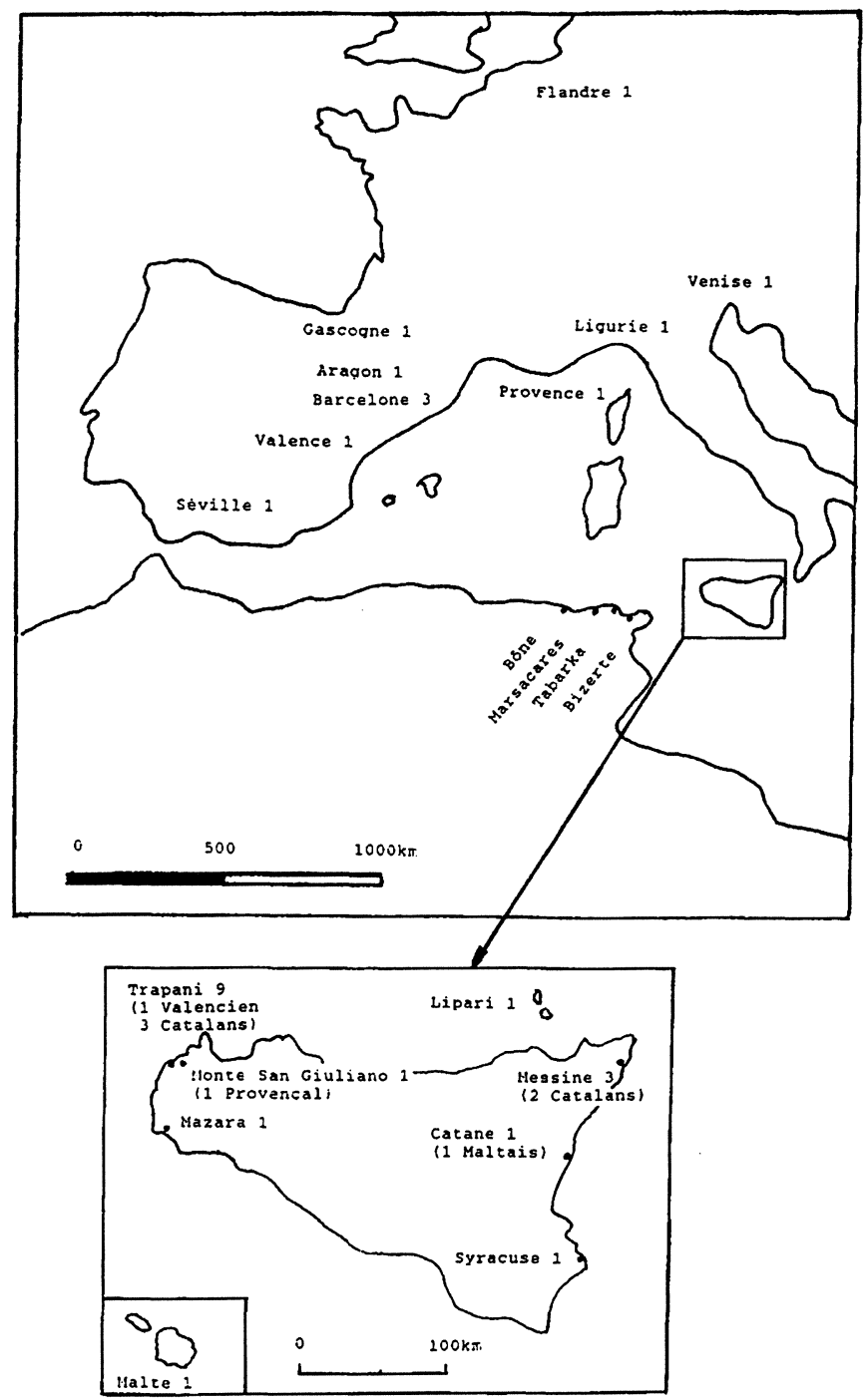

Légende: Messine 3 (2 catalans)

signifie que, parmi les trois hommes d'équipage qui résident à Messine, deux sont qualifiés de Catalans ou portent un nom catalan. 
grandes escales sur les principales routes maritimes du moment, Venise, Séville, la Gascogne et la Flandre.

Tableau n' 1 .

Origine géographique et lieu de résidence des pêcheurs

\begin{tabular}{|l|l|l|l|l|l|l||}
\hline & Total & résident & en Sicile & $\begin{array}{l}\text { résident } \\
\text { hors } \\
\text { Sicile }\end{array}$ & $\begin{array}{l}\text { Total des } \\
\text { non } \\
\text { Siciliens }\end{array}$ & $\begin{array}{l}\text { (dont } \\
\text { Catalans) }\end{array}$ \\
\hline & & Total & $\begin{array}{l}\text { (dont } \\
\text { étrangers) }\end{array}$ & & & \\
\hline Patrons & 28 & $23(82 \%)$ & $10(35 \%)$ & $5(19 \%)$ & $15(53 \%)$ & $14(50 \%)$ \\
\hline $\begin{array}{l}\text { Equipa- } \\
\text { ges }\end{array}$ & 29 & $16(55 \%)$ & $8(27 \%)$ & $13(45 \%)$ & $21(72 \%)$ & $11(38 \%)$ \\
\hline Total & 57 & $\begin{array}{l}39 \\
(68,5 \%)\end{array}$ & $\begin{array}{l}18 \\
(31,5 \%)\end{array}$ & $\begin{array}{l}18 \\
(31.5 \%)\end{array}$ & $36(63 \%)$ & $25(43 \%)$ \\
\hline
\end{tabular}

En définitive, les pêcheurs de corail qui se rendent à Tabarka entre 1446 et 1448 sont en majorité des non-Siciliens, parmi lesquels les Catalans prennent la première place, surtout chez les patrons de barque (tableau $\left.n^{\circ} 1\right)$.

On peut expliquer cette prépondérance catalane par le fait que Rafaël Vives a certainement contacté les patrons par l'intermédiaire du réseau des consulats catalans; d'autre part, les deux grandes régions productrices de corail les plus proches de Tabarka, la Sardaigne et la Sicile, font toutes deux partie de «l'empire méditerranéen» de la Couronne d'Aragon et les Catalans sont très actifs sur les bancs d'Alghero et de Trapani; en effet, depuis qu'en 1335, Pierre IV a réglementé la pêche du corail à Alghero, les Catalans en dominent la production ${ }^{35}$; de même, lorsque le banc de Trapani est découvert en 1418, ce sont encore les Catalans, souvent venus de Sardaigne, qui apportent leur expérience et permettent le développement de l'exploitation $^{36}$.

En revanche, le faible nombre des Siciliens et des Trapaniotes est étonnant, surtout chez les hommes d'équipage; les contrats étant tous rédigés

${ }^{35} \mathrm{~F}$. PODESTÀ, I Genovesi e le pescherie di corallo nei mari dell'isola di Sardegna, dans "Miscellanea di Storia Italiana", serie 3, VI-100, p. 16.

${ }^{36} \mathrm{G}$. BRESC-BAUTIER, Le corcail sicilien, op. cit., p. 183. 
à Trapani, on pouvait s'attendre à un fort recrutement de pêcheurs parmi la main d'œuvre locale; il n'en est rien. D'autre part, les équipages ne présentent aucune homogénéité, l'origine du patron ne présumant pas celle de ses associés ou de ses salariés; sur les quatre associés de Johannes Sagoya, un seul est trapaniote, deux sont barcelonais, le dernier venant de Syracuse $^{37}$; dans une autre barque, le patron trapaniote est associé à deux Provençaux et à un Valencien ${ }^{38}$; l'équipage d'un patron de Messine est plus homogène puisqu'il réunit deux hommes de la même cité et un Ligure ${ }^{39}$; en revanche, les neuf hommes recrutés comme salariés par Bartholomeus de Avillaneda sont tous des étrangers ${ }^{40}$; sans doute, la diversité d'origine des équipages doit-elle parfois être tempérée par le fait que certains étrangers sont des immigrants récents; l'un des Provençaux habite Monte San Giuliano et le Valencien est trapaniote d'adoption; mais ce n'est pas le cas des deux Barcelonais de Johannes Sagoya, ni des neuf hommes recrutés par Bartholomeus de Avillaneda.

Tenter d'expliquer cette faible participation sicilienne et trapaniote à l'exploitation du corail de Tabarka revient à poser un certain nombre d'interrogations; autant d'hypothèses qui, d'ailleurs, ne s'excluent pas l'une l'autre. Tout d'abord, il faut rappeler que, sur un total théorique de 125 hommes d'équipage, nous ne connaissons le nom et l'origine que de 29 d'entre eux et cet échantillon pourrait n'être pas représentatif. Néanmoins, des raisons structurelles et conjoncturelles peuvent être avancées. La Sicile manquerait-elle d'hommes et de pêcheurs de corail en particulier? Pourtant, les Catalans prépondérants au début de l'exploitation des gisements en 1418 semblent avoir progressivement cédé la place aux pêcheurs locaux ${ }^{41}$. Le salaire mensuel de 21 tari proposé aux hommes d'équipage, sans atteindre le niveau de ceux des maçons ou des bouchers, est nettement supérieur à celui d'un polisseur de corail et devrait attirer une main d'œuvre en quête

\footnotetext{
${ }^{37}$ Durduglia, 8606, 14 mars $1447, f^{\circ} 125 \mathrm{v}$.

${ }^{38}$ Miciletto, 8593, 15 avril, 1447, $f^{\circ} 78 \mathrm{v}$.

${ }^{39}$ Milo, 8626, 17 juin 1447, $\mathrm{f}^{\circ} 306 \mathrm{r}$.

${ }^{40}$ Les villes ou régions d'origine de ces marins sont: l'Aragon, la Flandre, la Gascogne, Barcelone, Messine, Séville, Valence, Venise; l'origine du neuvième marin est inconnue car le début de l'acte le concernant est illisible; références de ces actes, cf. note 30.

${ }^{4 \prime}$ G. Bresc-BAutier, Le corail sicilien, op. cit., p. 183.
} 
de travail $^{42}$. Faut-il penser que les Trapaniotes jugent l'expédition à Tabarka particulièrement dangereuse? Certes le danger est réel; en 1448, un équipage est retenu prisonnier ${ }^{43}$; de son côte. Bartholomeus de Avillaneda fait son testament quelques jours avant son départ ${ }^{44}$, mais la démarche est coutumière et les risques encourus paraissent inférieurs à ceux de la guerre de course pour laquelle les Siciliens montrent des aptitudes certaines; en outre, les années d'exploitation du corail de Tabarka correspondent à une période de paix et d'intenses échanges commerciaux entre la Sicile et le royaume hafside ${ }^{45}$. Faut-il alors croire les pêcheurs sardes qui accusent Rafaël Vives de prélever une part trop importante du corail pêché, décourageant ainsi les pêcheurs potentiels? C'est possible. En définitive, le manque d'hommes en Sicile - trois mois sont nécessaires pour recruter l'équipage du patron Avillaneda- et les conditions financières médiocres offertes par le marchand catalan ont attiré une main d'œuvre étrangère ou sans attache géographique, allant de port en port à la recherche d'une opportunité.

\section{Une organisation légère et peu centralisée}

L'organisation mise en place par Rafaël Vives pour l'exploitation du corail de Tabarka demeure presque totalement inconnue, mais les rares indices que l'on peut glaner montrent une structure bien différente de celle que Clemente Cicero établira à Marsacares après 1451; En effet. l'éloignement des côtes maghrébines de la Ligurie oblige les frères Cicero à mettre en place une structure très centralisée leur donnant la maîtrise de l'ensemble des opération et leur assurant la majeure part des bénéfices; tandis que l'un des frères recrute patrons et pêcheurs en Ligurie, pourvoit aux approvisionnement et financements nécessaires, Clemente, qui réside à Marsacares,

\footnotetext{
${ }^{42} 13$ tari par mois pour un artisan ad rotundandum curallum: AST, notaire Giovanni Castiglione. 8708. 4 juillet 1455. $\mathrm{f}^{\circ} 91 \mathrm{r}$ : au début du siècle les maçons et les bouchers reçoivent un salaire mensuel de 30 à 40 tari: H. BRESC. Un monde méditerranéen. op. cit.. I. p. 213.

${ }^{43}$ Milo, 8628, 15 février 1448, $\mathrm{f}^{\circ} 176 \mathrm{r}$

${ }^{4}$ Miciletto, 8593, 19 avril 1447, $\mathrm{f}^{\circ} 81 \mathrm{r}$.

${ }^{45} \mathrm{D}$ après une étude (à paraître) que jentreprends actuellement sur les relations entre la Sicile et le Maghreb à partir de sources notariées des archives de Trapani, la période d'intenses échanges commerciaux s'achève brutalement dans les années 1450-1451: ensuite c'est la course qui domine jusqu à la trêve signée en 1473.
} 
dirige les opérations en véritable entrepreneur, répartissant les lieux de pêche, approvisionnant les barques et les hommes en matériel de pêche et en nourriture, tenant dans ses livres les comptes de chaque barque et de chaque marin, et, surtout, se réservant le monopole de l'acquisition du corail ${ }^{46}$.

Rien de tel avec Rafaël Vives, dont le rôle semble se réduire au prélèvement de sa part de corail; l'organisation de son exploitation du corail de Tabarka ressemble en fait à toutes les exploitations dans lesquelles les Catalans sont impliqués, en particulier celles de Sardaigne et de Sicile; les pêcheurs doivent, tout d'abord, obtenir une licence royale pour pêcher le corail de Tabarka comme leurs collègues qui pêchent à Alghero ou Trapani $^{47}$; ensuite, ils sont responsables de leur approvisionnement en nourriture et en matériel de pêche et doivent trouver les financements nécessaires; enfin, ils assurent eux-mêmes la vente de leur part de corail en la réservant à l'avance à un acheteur unique ou en la vendant au meilleur prix possible au retour de la campagne; Rafaël Vives n'exerce donc aucun monopole sur la vente du corail brut ${ }^{+1}$.

Sur place, à Tabarka, Rafaël Vives a dû nécessairement exercer son contrôle sur le corail pêché pour en prélever sa part et empêcher la fraude; on ignore s'il a délégué cette charge, mais, très certainement, il n'a pu, comme les Génois de Marsacares, disposer d'une barque de surveillance, le leudo goardie, ni d'un corps de garde ${ }^{49}$. Rafaël Vives a-t-il, d'autre part, fait édifier sur l'île des bâtiments destinés à entreposer le matériel de pêche ou stocker le corail? Ces éventuelles constructions, ainsi que les traces de l'exploitation catalane, ont naturellement été recouvertes par les occupations successives, en particulier par le comptoir génois du XVI" siècle, mais les

\footnotetext{
${ }^{46}$ Sur l'organisation de l'exploitation du corail de Marsacares, se reporter à: P. Gourdin, Émigrer au $X V^{\prime \prime}$ siècle. op. cit. note 3.

${ }^{47}$ Autorisation de se rendre à Tabarka: Bibliothèque Fardeliana de Trapani. Atti del Senato, Registro di Lettere. II, 8 ou 9 décembre 1447. $f^{\circ} 326 \mathrm{v} 327 \mathrm{r}$; en Sardaigne. les pêcheurs doivent obtenir la licence et payer la taxe à la douane d'Alghero: F. PODESTÀ, I Genovesi e le pescherie di corallo. op. cit., p. 184.

${ }^{48}$ En effet. dans un litige opposant Rafaël Vives à trois patrons catalans de Cagliari, ce n’est pas le fait de vendre du corail qui est reproché aux patrons. mais de vendre du corail qui appartient à Rafaël: les arbitres nommés pour régler cette affaire donnent raison à Rafaël: Milo. 8626, 10 et 18 juillet 1446 . $f^{\circ} 329 \mathrm{r}$ à $330 \mathrm{v}$; il s"agit, en fait, du conflit portant sur la part de corail pêché qui revient aux patrons: nayant obtenu satisfaction dans cet arbitrage qui est rendu dans la loge des Catalans de Trapani, les pêcheurs sardes tentent leur chance en adressant leur réclamation à Barcelone: d'où la lettre des conseillers de Cagliari à ceux de Barcelone du 20 novembre 1446 et la réponse du 14 décembre 1446 (références note 4).

${ }^{49} \mathrm{P}$. Gourdin, Émigrer au XV' siècle. op. cit., I, p. 584.
} 
fouilles actuellement conduites dans l'île n'ont pas permis d'en repérer l'existence ${ }^{50}$; la présence catalane et sicilienne qui n'a duré qu'un peu plus de deux ans avec une occupation discontinue des lieux n'a pu laisser que des traces extrêmement ténues; si Rafaël Vives édifia des bâtiments dans l'île, ceux-ci furent construits en matériaux légers et disparurent rapidement; à la fin du siècle, le Turc Piri Reis, qui accompagne son oncle dans ses expéditions en Méditerranée occidentale, ne signale aucune construction sur l'île alors qu'il remarque la forteresse en ruine de la terre ferme ${ }^{51}$; et lorsqu'il est question, sous Charles Quint, de reprendre l'exploitation du corail de Tabarka, il est aussitôt envisagé d'y construire une tour ${ }^{52}$.

\section{LA CESSION DE LA PÊCHE DU CORAIL AUX EUROPÉENS}

La décision du souverain hafside de céder l'exploitation du corail de son royaume à Rafaël Vives est lourde de conséquences; la pêche du corail maghrébin restera désormais entre les mains des chrétiens, Catalans et Siciliens en 1446, Génois en 1451 et Français à la fin du XVIII ${ }^{\mathrm{c}}$ siècle $^{53}$,

\footnotetext{
${ }^{50}$ Depuis 1987. la Mission archéologique de Tabarka entreprend des recherches sur l'île de Tabarka: cette mission tuniso-française associe l'Institut National du Patrimoine de Tunis (ex Institut National d’Archéologie et d'Art), l’École française de Rome et le Ministère français des Affaires Etrangères; les premiers résultat de cette recherche paraitront dans: P. GoURDIN. Tabarka. Histoire et archéologie d'un comptoir européen en terre africaine.

${ }^{51} \mathrm{C}$ 'est en 1487 que le corsaire turc Kemal Reis débute ses expéditions en Méditerranée occidentale: son neveu, le futur grand amiral et cartographe. rédige plusieurs années après une description des côtes du Maghreb; il décrit Tabarka en ces termes: "La forteresse de Tabarka (est) depuis longtemps en ruine: devant cette forteresse, il y a une petite ile qui est un bon port pour les petits navires et les galères: les navires qui s’amarrent à l'île jettent l'ancre suivant le vent, car tout point de l’île convient; les navires qui entrent dans le détroit de l’île ne peuvent le faire que par l'ouest. car de ce côte le passage est large et sa profondeur est de quatre ou cinc brasses»: R. Mantran, La description des côtes de Tunisie dans le Kitâb-i Bahriye de Piri Reis, dans "Revue de l'Occident musulman et de la Méditerranée" (1977-2), pp. 223-224.

52Archivio di Stato de Parme. Carte Gonzaga 42-4, Instruttione particolar di quello che s'ho da referire al Ill.mo S.or Duca di Sabbiotta sopra il particolar de la pesca del corallo, $f^{\circ} 142 r$. "Sara necessario, accio che i pescatori potessero andar sicuramente a pescar senza sospetto o pericolo desser presi da corsari far fabricar in detta isola di Tabarca una torre». lauteur du rapport est Ferrante Gonzaga, viceroi de Sicile (rapport annexé à la correspondance du vice-roi de Sicile adressée à l'empereur: Registro di cose di guerra 1540-1543).

${ }^{53}$ Les Français sont présents à Marsacares dès le XVI' siècle et un peu plus tard au Cap Nègre: mais leurs efforts pour s̈implanter à Tabarka ne réussiront qu'en 1781: P. MASSON, Histoire des établissements et du commerce français dans l'Afrique barbaresque (1560-1793). Paris, 1903: Les compagnies du corail, Paris-Marseille, 1908.
} 
excluant totalement les pêcheurs locaux. Plusieurs hypothèses peuvent être avancées pour expliquer cette cession.

Il faut remarquer, en premier lieu que les bénéficiaires, Rafaël Vives et Clemente Cicero présentent certaines ressemblances; tous deux sont des hommes d'affaires fortement et durablement implantés à Tunis; les activités de Rafaël dans la capitale hafside peuvent être suivies des années 1440 aux années $1470^{54}$; en 1472 , il est chargé par le vice-roi de Sicile de négocier le rachat de 500 captifs chrétiens détenus dans le royaume hafside ${ }^{55}$; déjà, en 1457, il avait accompagné en Sicile un représentant du souverain hafside venu recenser les esclaves maures insolvables ${ }^{56}$. Clemente Cicero joue également un rôle officiel; son nom figure parmi les témoins du traité signé à Tunis entre la Commune de Gênes et Abu Fares en 1433; on le retrouve consul des marchands génois de mars 1450 à mars 1452, puis négociateur, actionnaire principal et gouverneur pendant une vingtaine d'années de la compagnie génoise qui exploite le corail de Marsacares, résidant soit à Tunis soit sur les lieux de production; lorsqu'il quitte la direction de la compagnie, il demeure certainement dans la capitale hafside où il possède un funduq qui conserve son nom après sa mort ${ }^{57}$.

Une telle implantation entraîne non seulement une bonne connaissance des lieux, des usages et, sans doute, de la langue, mais également une certaine familiarité avec le souverain; l'un et l'autre participent à des discussions diplomatiques; ils sont également sollicités par des compatriotes, pour obtenir, en dehors des circuits officiels, la libération de marchands chrétiens emprisonnés par les autorités hafsides ${ }^{58}$. En 1447, au moment de l'exploitation du corail de Tabarka, Rafaël Vives est particulièrement bien en cour; il n'hésite pas à rejoindre le souverain hafside dans son «camp»

${ }^{54}$ C. Carrère. Barcelone centre économique 1380-1462. I. Paris. 1967. p. 148 : B. DOUMERC. Le consulat rénitien de Tunis. dans "Cahiers de Tunisie". 155-156. p. 461.

${ }^{55} \mathrm{~L}$. DE MAS LATRIE. Traités de paix et de commerce. op. cit., II, p. 124. lettre du 20 novembre 1472

${ }^{56} \mathrm{H}$. BRESC, Un monde méditerranéen, op. cit., I, p. 448, n. 19

${ }^{57}$ En 1502, il existe encore un funduy Cicero à Tunis: Archivio di Stato de Gênes (ASG), notaire Agostino Testa, 6 janvier 1502.

${ }^{58}$ Clemente Cicero intervient auprès de Othman pour faire libérer deux Génois emprisonnés à Sfax en 1453: ASG. Busta Africa, 2774c, document publié dans : E. MARENGO, Genov'a e Tunisi, 1388-1515, dans "Atti della Società ligure di Storia Patria". XXXII (1901), p. 156; Rafaël Vives fait de même en 1447 pour faire libérer deux Barcelonais, les frères Dez Pla: C. CARRĖRE, Barcelone centre économique, op. cit., I, p. 148. 
pour négocier de telles libérations tout en prévenant ses compatriotes que les tractations avec les Maures sont toujours longues ${ }^{59}$; au reste, il dispose vis à vis du souverain d'un important moyen de pression puisque ce dernier lui doit la somme de 7.000 doubles d'or ${ }^{60}$; sachant qu'en 1451, Clemente Cicero obtiendra la concession de la pêche du corail dans les mers de Barbarie contre le payement annuel de 2.000 doubles, on peut raisonnablement supposer que la cession de ce droit à Rafaël Vives fut, pour le roi hafside, un moyen commode d'annuler tout ou partie de sa dette.

L'utilisation par les Hafsides de la concession ou de l'affermage n'est pas nouvelle; déjà, au XIII' siècle, la gabelle des vin de Tunis est affermée à des Européens et des Génois deviendront en 1483 fermiers de la douane de Bougie ${ }^{61}$; ces rentrées fiscales restent toutefois modestes en comparaison de la taxe sur les transactions que les marchands étrangers doivent acquitter sur les ventes et achats qu'ils effectuent à l'intérieur du territoire hafside ${ }^{62}$. Othman encourage ces activités car elles contribuent fortement au budget de son état; d'autre part, il n'hésite pas à se faire commerçant lui-même, achetant des parts de bateaux, négociant des achats d'armes ${ }^{63}$; en effet, au delà du simple appât du gain, il faut voir dans la politique d'ouverture pratiquée par Abu Fares, puis par Othman, auprès des différents états chrétiens, la volonté de financer une armée moderne pour combattre toute velléité d'indépendance des princes hafsides et des tribus arabes, et, éventuellement, pour revendiquer l'hégémonie au Maghreb; il n'est pas

\footnotetext{
${ }^{59}$ "Los moros son naturalment lonch de conclusio» cité par C. CARRĖRE, op. cit., I, p. 148

${ }^{60}$ "Lo rey de Tuniç me era tengut de 7000 dobles" affirme Rafaël Vives dans une lettre aux conseillers de Barcelone: C. CARRÈRE. Ibidem.

${ }^{61} \mathrm{R}$. Brunschvig, La Berbérie orientale sous les Hafsides des origines à la fin du $\mathrm{XV}$ siècle. II, Paris, 1947, p. 71.

"Cette taxe n'est pas véritablement un droit de douane puisque les marchandises importées et non vendues peuvent être réexportées sans acquitter la taxe; le taux normal est de $10 \%$.

${ }^{6.3}$ Dans une lettre du 5 février 1452 adressée à la Commune de Gênes, Othman fait part d'une commande d'armes; le même document révèle qu'il est propriétaire du quart d'une nave génoise: ASG, Diversorum, année 1453-1464, $\mathrm{n}^{\circ}$ 338, document publié dans M. AMARI, Nuovi Ricordi Arabi sulla storia di Genova, dans "Atti della Società ligure di Storia Patria", V (1873), pp. 617-623; des marchandises diverses appartenant à Othman sont transportées de Tripoli à Tunis dans la nave du Génois Vinciguera Vivaldi: ASG, notaire Baromeo Risso, F. 5, $n^{\circ} 78$. 20 juin 1457.
} 
étonnant, dans ces conditions, de constater la simultanéité d'une croissance du commerce international et du renforcement du pouvoir central ${ }^{64}$.

En 1446, Othman a donc de bonnes raisons d'accorder la concession de la pêche du corail à Rafaël Vives; il est son débiteur et la trêve avec Alphonse d'Aragon place les Catalans dans une situation privilégiée; pourtant l'argument financier semble insuffisant; Othman aurait pu faire payer une licence à des pêcheurs locaux, organiser à son profit la production et se réserver la totalités des bénéfices; il n'a pas tenté l'entreprise; ne faut-il pas penser que cela était impossible faute de pêcheurs locaux?

En effet, il n'est pas certain que l'exploitation du corail maghrébin décrite au $\mathrm{XII}^{\mathrm{e}}$ siècle par Idrissi ${ }^{65}$ se soit maintenue jusqu'au $\mathrm{XV}^{\mathrm{e}}$ siècle; certes, au XIV ${ }^{\mathrm{e}}$ siècle, Abulfeda cite Marsacares comme centre de pêche et compare la qualité de son corail avec celle du corail qui est récolté dans la région de Bône; mais cela ne signifie nullement que l'on pêche encore le corail à son époque car il tire un grand nombre de ses informations du livre d'Idrissi $^{66}$. D'autre part, le travail du corail, en particulier son polissage et la fabrication de boutons ou d'objets plus élaborés, attestés dès le $\mathrm{XI}^{\mathrm{e}}$ siècle par les documents de le Genizah du Caire ${ }^{67}$, ne sont plus connus dans le royaume hafside au temps de Rafaël Vives car une ordonnance des conseillers de Barcelone, datée du 2 juin 1447, interdit aux ressortissants catalans de se rendre en terre des Maures pour y travailler le corail, enseigner leur savoir-faire, ou encore, apporter les instruments nécessaires $^{68}$. Les courants d'échange entre la Sicile et l'Ifrikiya confirment les

\footnotetext{
${ }^{64}$ De 1435 à 1452, Othman doit combattre son oncle Abu'l Hasan qui, de sa principauté de Constantine et de Bougie, se comporte en souverain, battant même parfois monnaie: $\mathrm{R}$. BRUNSCHVIG, La Berbérie orientale, op. cit., I, 1940, p. 243. 141.

${ }^{65}$ Al IDRISSI, Le Maghreb au V' siècle de l'Hégire, trad. M. Hadj Sadok, Paris, 1983, p.

${ }^{66}$ «Marsacares où se fait la pêche du corail... "; " Dans le voisinage de Bône, on pêche du corail qui n'est pas de la même qualité que celui de Mars'alkharaz": Géographie d'Abulfeda, trad. M. Reynaud, II, Paris, 1848. pp. 191, 194.

${ }^{67}$ S. D. GoITEIN, Letters of Medieval Jewish Traders, Princeton, 1973, p. 280; Lorigine du corail travaillé n’est pas indiquée, mais la provenance maghrébine est probable, au moins partiellement.

${ }^{68}{ } \mathrm{~N} \mathrm{Ni}$ sia licit ni permes anar en terra de moros o d altres infels per obrar ni fer obrar corals ni exercitar en aquelles parts ni mostrar lo dit offici a qualsevol moro o infel ni encara apporta ni trasmetre hi algunes artilleres o instruments del dit offici; e aço, sots ban de perdre la vida e los bens... "Archives de la ville de Barcelone, crides originals, document publié par L. CAMOS Y CABRUJA, Referencias documentales en torno al tráfico del coral en Barcelona en el siglo XV, dans "Boletín de la Real Academia de Buenas Letras de Barcelona" (1946), p. 189.
} 
instructions de cette ordonnance: tandis que Rafaël Vives et Clemente Cicero récoltent le corail brut sur les côtes hafsides, les flux de corail travaillé se dirigent toujours de Sicile vers le Maghreb ${ }^{69}$.

Les raisons de l'arrêt de la production de corail maghrébin, probablement dès le XIII' siècle, sont multiples: Insécurité qui règne sur les lieux de production; Marsacares est pillé par Roger de Lauria en 1287; incessante guerre de course au XIV $\mathrm{XV}^{*}$ siècle; guerre civile en Ifrikiya; par trois fois l'unité du royaume est combattue par les princes hafsides de l'ouest, en particulier ceux de Bougie et de Bône dont les territoires atteignent les zones de production. Mais des raisons moins locales peuvent également être avancées; la demande globale de corail s'est peut-être raréfiée, en particulier celle du principal marché, l'Egypte et l'Orient; ou, plus vraisemblablement, cette demande est désormais satisfaite par les producteurs du nord de la Méditerranée occidentale, ceux-là mêmes qui contrôlent de plus en plus les principales routes maritimes de Méditerranée.

A cet égard, l'analogie avec la situation sicilienne est frappante. G. Bresc-Bautier a bien montré que c'est également Idrissi qui parle, au milieu du XII ${ }^{e}$ siècle, du «corail de première qualité» de la région de Trapani, information reprise par des auteurs plus tardifs comme Al-Dimisqi, au début $\mathrm{du}^{\mathrm{XIV}} \mathrm{XV}^{\mathrm{C}}$ siècle, tandis que les archives siciliennes n'attestent une production locale chrétienne qu'en 1388, à l'occasion de la venue de pêcheurs provençaux et surtout, à partir de 1418, au moment de l'exploitation du gisement de Trapani par les Catalans ${ }^{70}$; comme les maghrébins ${ }^{71}$, les Siciliens ont abandonné la pêche du corail après le XII" siècle. En revanche, l'exploitation du corail est attestée de façon continue dans le nord de la Méditerranée occidentale, depuis le milieu du $\mathrm{XI}^{\mathrm{e}}$ siècle en Catalogne ${ }^{72}$, à partir de 1154 à Portofino en Ligurie $^{73}$ et depuis la première moitié du

\footnotetext{
${ }^{69}$ Transport de corail travaillé de Sicile vers le Maghreb: Durduglia, 8626, $\mathrm{f}^{\circ} 78 \mathrm{v}, 13$ décembre 1446; $\mathrm{f}^{\circ} 120 \mathrm{v}, 6$ mars 1447: transport de patenôtres de corail vers Tripoli de Barbarie: AST, notaire Giovanni Forziano, 8741, $f^{\circ} 143 \mathrm{v}, 17$ mai 1451

${ }^{70} \mathrm{G}$. BRESC-BAuTIER, Le corail sicilien, op. cit., pp. 182-183.

${ }^{71} \mathrm{~L}$ exploitation du corail du site marocain de Ceuta est également abandonnée au XIII siècle: H. Ferhat, Sabta des origines au XIV' siècle, Rabat, 1993, pp. 278-279.

${ }^{72} \mathrm{G}$. BRESC-BAUTIER, Le corcill sicilien, op. cit.. p. 183.

${ }^{73}$ O. PASTINE, Liguri pescatori di corallo, dans "Giornale storico e lettererio della Liguria", VII (1931), p. 171
} 
XIIIe siècle en Provence ${ }^{74}$; en outre, tout en poursuivant l'exploitation de leurs bancs locaux de corail, ces pêcheurs accompagnent l'expansion de leur cité d'origine en direction du sud de la Méditerranée; ainsi les Marseillais sont présents en Sardaigne dès la deuxième moitié du XIII' ${ }^{\circ}$ siècle ${ }^{75}$ et dans le royaume de Naples à partir de $1277^{76}$ et les Provençaux apparaissent en Sicile en $1388^{77}$; les Catalans remplacent les Marseillais en Sardaigne au

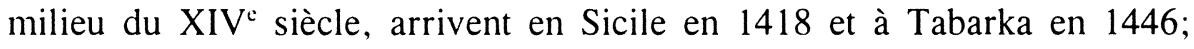
quant aux Génois et Ligures, leur présence à Bosa en Sardaigne dans la première moitié du XIV $\mathrm{XI}^{*}$ siècle est contrariée par l'arrivée des Catalans ${ }^{78}$ et ce n'est que dans la deuxième moitié du $X V^{c}$ siècle qu'ils étendent leur influence au Maghreb, en Sardaigne et en Corse, en contrôlant les gisement de Marsacares en 1451, d'Alghero en $1466^{79}$ et de Bonifacio dans le dernier quart du siècle ${ }^{80}$.

Dans cette lutte pour le contrôle des meilleurs coraux, les pêcheurs du nord de la Méditerranée disposent d'atouts décisifs; contrairement aux pêcheurs de Sicile et du Maghreb, ils n'ont pas abandonné leur activité et ont conservé leur savoir-faire au point d'être à l'origine de toutes les nouvelles exploitations postérieures au milieu du XIII' siècle; en outre, cette activité est stimulée par les commerçants de leur cité qui exportent leur production le long des routes maritimes qu'ils contrôlent; en effet, autre analogie entre la Sicile et le Maghreb, ce sont les grands marchands du nord de la Méditerranée occidentale qui contrôlent les exportation de leur corail; si les Siciliens travaillent leur corail au $\mathrm{XV}^{\mathrm{c}}$ siècle, ainsi que le corail de Tabarka au temps de Rafaël Vives, ce sont les marchands catalans qui le portent en

\footnotetext{
${ }^{74}$ L. Bl.anCARD. Documents inédits sur le commerce de Marseille au Moyen Age. I. Marseille. 1885. p. 18.

${ }^{75}$ G. I a avergne. La pêche et le commerce du corcil ì Marseille, op. cit. p. 200. n. 25.

${ }^{76} L$ 'ordonnance du roi Charles ler d’Anjou du 25 février 1277 accorde aux pêcheurs marseillais et provençaux le droit de pèche dans le royaume de Naples: document publié dans G. TESCIONE. Italiani alla pesca di corallo ed egemonia marittima nel Mediterraneos, Naples, 1940. document $n^{\circ} 1$. pp. 281-282.

${ }^{77}$ G. BResc-BaUtier, Le corcil sicilien. op. cit. p. 183.

${ }^{78} \mathrm{~F}$. PODESTA, I Genovesi e le pescherie di corallo, op. cit. p. 15.

${ }^{70}$ Ibidem. p. 16 : J. HeERs. Gênes au XV siècle. Activités économiques et problèmes sociculx. Paris, 1961, p. 427.

${ }^{80}$ ASG, notaire Battista Parisola, F 3. n 168 . $1^{\text {cr }}$ octobre 1477.
} 
Orient ${ }^{81}$ et, dans la deuxième moitié du XVe siècle, le corail de Marsacares est vendu par les Génois en Ligurie et par les Vénitiens en Orient ${ }^{82}$.

\section{CONCLUSION}

La première exploitation du corail maghrébin par les Européens fut probablement un échec; c'est, du moins, ce que suggère sa courte durée. Rafaël Vives n'avait sans doute pas les moyens, ni la volonté d'organiser une structure lui laissant la majeure partie du bénéfice sur la pêche du corail, préférant se contenter de prélever, pour son propre compte, un tiers de la production; peut-être a-t-il simplement voulu, par ce moyen, annuler la dette du souverain hafside à son égard; encore n'est-il pas certain qu'il a pu, dans un temps aussi court, récupérer les 7.000 doubles dus par Othman et l'on comprend aisément son refus d'abaisser cette part du tiers au dixième du corail pêché ${ }^{83}$.

En 1451, lorsque Clemente Cicero reprend la concession de la pêche du corail, l'heure des Génois a sonné; en effet, le retrait de Rafaël Vives est suivi par l'arrêt presque total des échanges commerciaux entre la Sicile et l'Ifrikiya pendant une vingtaine d'années et marque le déclin de la présence catalane au Maghreb.

En mettant en concurrence les deux grandes nations marchandes du nord de la Méditerranée, Othman a pu obtenir des avantages immédiats en s'assurant des rentrées régulières; en revanche, sa décision de faire appel à

\footnotetext{
${ }^{81} \mathrm{G}$. Bresc-BAUTIER, Le corcill sicilien. op. (it. p. 188.

${ }^{82}$ P. Gourdin. Emigrer al XVe siècle, op. cit. II. p. 159; d’autre part. le ler décembre 1469. les actionnaires de la compagnie génoise qui exploite le corail de Marsacares s'engagent à livrer 550 cantares de corail pendant six ans à un groupe de marchands vénitiens au prix de 50 ducats le cantare: Archivio di Stato de Venise. Cancelleria Inferiore. B 27, notaire Belleto. $\mathrm{f}^{\circ} 6 \mathrm{v}$ à $8 \mathrm{v}$ (document aimablement fourni par B. Doumerc): sur le rôle des Vénitiens dans la vente du corail maghrébin en Orient pendant la deuxième moitié du XV siècle. se reporter à B. DOUMERC. Le consulat vénitien de Tumis. op. cit.. p. 457.

${ }^{83} \mathrm{C}$ 'est ce que montre un rapide calcul : le rotulus de corail brut est acheté 4 tari à Trapani et le double hafside vaut 8 tari : pour récupérer 2000 doubles par an. somme qui sera demandée aux frères Cicero dans la concession de 1451. Rafaël doit disposer de 40 cantares de corail, ce qui correspond à 120 cantares pêchés si le prélèvement est de un tiers: soit 4,8 cantares par barque s'il y a 25 barques ou 2.4 cantares par barque s il y a 50 barques: rappelons que les pêcheurs qui se rendent à Tabarka restent habituellement de deux à cing mois sur les lieux de pêche. Par comparaison. en 1469. les Génois de Marsacares s'engagent à vendre un minimum de 550 cantares de corail à un groupe d'acheteurs vénitiens, alors que l'on sait par ailleurs, que 70 à 80 barques sont à demeure à Marsacares et que chacune d'entre elles doit pêcher un minimum de 8 cantares dans l'année: P. Gourdin. Emigrer al XV siècle. op. cit., II. p. 146.
} 
des Européens pour exploiter le corail de ses côtes est lourde de conséquences à long terme; elle représente un pas important dans la progression des intérêts européens en Ifrikiya.

\section{RÉSUMÉ}

En 1446, le souverain hafside Othman cède l'exploitation du corail de son royaume à un Catalan vivant à Tunis. Rafaël Vives, qui fait venir à Tabarka des corailleurs venant de Sicile et de Sardaigne, en majorité des Catalans expatriés. Cette exploitation qui ne dure que quelques années est l'une des dernières manifestations du dynamisme catalan en Méditerranée occidentale. contré par l'expansion génoise à partir du milieu du XV" siècle. Pour le royaume hafside. cette première intervention marque le début de l'emprise définitive des Européens (Catalans. puis Génois et Provençaux) dans l'exploitation corail maghrébin. qui se prolongera jusqu à la période contemporaine malgré des traditions locales fort anciennes.

\section{SUMMARY}

in 1446 Hafsid king Othman assigns the coral exploitation of north African coasts (1) a Catalan merchant living in Tunis. Rafaël Vives. The fishing grounds lay off Tabarka island and the fishers, mostly of Catalan origin. come from Sicily and Sardinia. This two years exploitation is one of the last evidence of the Catalan vitality in north Africa, harmed by genoese expansion in the second part of the XVth century. In the Hafsid kingdom this Catalan company is the first of many European settlements (Catalan, Genoese. Provençal and French) involved in the exploitation of coral in Maghreb until the XIXth century, in spite of ancient local traditions. 